\section{Polarized Thermal Emission from Narrow Tungsten Filaments}

From experimental work carried out by Fresnel many years ago and a theoretical investigation made by $\mathbf{K}$. Schwarzschild ${ }^{1}$, it is well known that light transmitted through a narrow slit is more or less polarized. Though conditions are rather different in the case of thermal emission from a narrow filament the idea has occurred to $m e$ that polarized emission may be obtained in this way in addition to inclinedsurface-layer-effects described by Worthing ${ }^{2}$ and others (Arago effect ${ }^{3}$ ). Through the courtesy of Dr. G. Siljeholm of the Lumalampan Aktiebolag in Stockholm I have been able to test recently some filaments no thicker than $4 \cdot 8 \mu$. The experiments were carried out at the Swedish Solar Observatory in Anacapri, and a polarization as high as 28 per cent has been found in the red. The plane of vibration (electric vector) is perpendicular to the direction of the filament. Considering the fact that the $4 \cdot 8 \mu$ filament had not been polished as in the case of Worthing's measurements, the high value obtained in the red (28 per cent instead of 19 per cent found by Worthing) gives some indication that a 'slot-aerial' effect might be present. To some extent this view is supported by measurements of a $6 \cdot 5 \mu$ filament showing already a smaller degree of polarization than a polished broad filament. Furthermore, when examining with a Savart polariscope the interference fringes appearing in highly magnified images, it has been found that the polarization appears to be nearly the same in the central part of the wire as at the limb. Though the resolving power may have been insufficient for separating the regions clearly, it seems hard to explain the observation by means of the Arago effect alone.

By using different colour screens I have been able to establish an increase of polarization with wavelength. The determinations are presented in Table 1. The effective wave-length $\lambda$ transmitted through the filters has been estimated with a spectroscope. The degree of polarization was measured with a Cornu polarimeter, except in the case of infra-red radiation for which photographic observations were made. Special precautions were taken to avoid polarization effects due to reflexion in the glass tube, etc.

In Table 1 the quantity $\frac{d}{\lambda}$ is given as well as $P \frac{d}{\lambda}$, $d$ being the thickness of the wire. As is evident from the last column $P_{\bar{\lambda}}^{d}$ is remarkably constant and approximates to 2 . ${ }_{\lambda}^{d}$ using dots for $d=6.5 \mu$ and crosses for $d=4.8 \mu$.

In Fig. 1 the degree of polarization is plotted against The dots follow closely a straight line. All values follow closely the hyperbola $P_{\bar{\lambda}}^{d}=2$ presented as a dashed curve. When using instead $1 / \lambda$ as abscissa the crosses and the dots give different relationships. This indicates again that an aerial effect may be present. The

\begin{tabular}{|c|c|c|c|}
\hline$d(\mu)$ & $\lambda(\mu)$ & $\begin{array}{c}\text { Table } \\
d \\
\bar{\lambda}\end{array}$ & $P$ \\
\hline 6.5 & $\left\{\begin{array}{l}0.48 \\
0.55 \\
0.64 \\
0.66 \\
0.82\end{array}\right.$ & $\begin{array}{r}13 \cdot 5 \\
11 \cdot 8 \\
10 \cdot 2 \\
9 \cdot 8 \\
7 \cdot 9\end{array}$ & $\begin{array}{l}0.14 \pm 0.02 \\
0.16 \pm 0.01 \\
0.16 \pm 0.02 \\
0.19 \pm 0.02 \\
0.22 \pm 0.02\end{array}$ \\
\hline $4 \cdot 8$ & $\left\{\begin{array}{l}0.48 \\
0.66\end{array}\right.$ & $\begin{array}{r}10 \cdot 0 \\
7 \cdot 2\end{array}$ & $\begin{array}{l}0.22 \pm 0.02 \\
0.28 \pm 0.02\end{array}$ \\
\hline
\end{tabular}

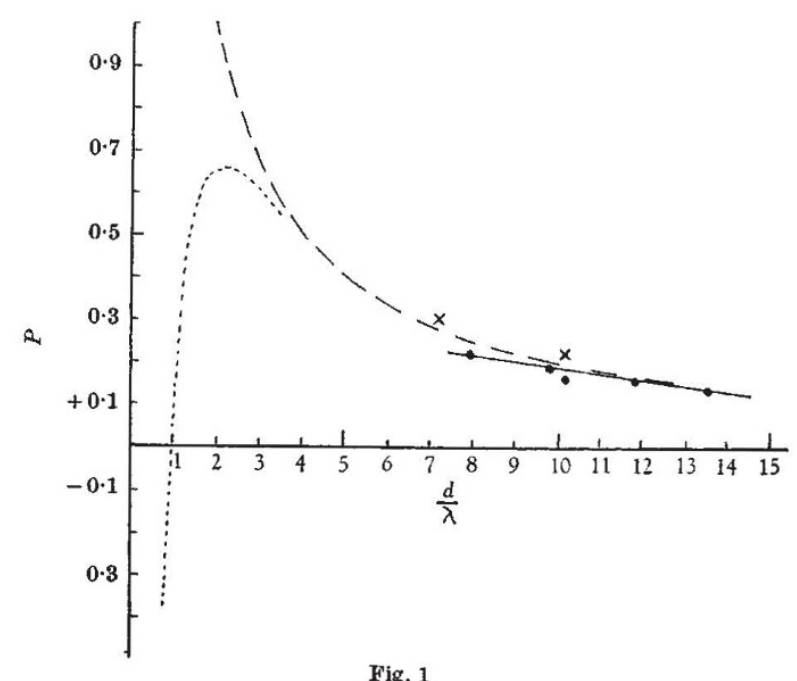

probable behaviour close to $\frac{d}{\lambda}=1$ has been indicated by the dotted curve by taking into account the phenomena produced by a narrow slit. If the relation $P_{\bar{\lambda}}^{d}=2$ is valid also for smaller values of $\frac{d}{\lambda}$ than those examined here, the highest polarization would be expected when the thickness of the filament is about twice the wave-length of light.

Preparations are being made for extending the measurements to narrower filaments. It is planned to ascertain also whether narrow bolometer filaments may be used for the purpose of analysing polarization in the infra-red.

Stazione Astrofisica Svedese,

YNGVE ÖHMAN Anacapri Italy.

${ }^{1}$ Schwarzschild, K., Math. Ann., 55, 177 (1901). Compare aiso similar effects produced in gratings (Riecke, Lehrbuch der Physik, 2, 375;

Worthing, A. G., J. Opt. Soc. Amer., 13, 635 (1926).

3 Öhman. Y., Polarization Measurements in Astronomy (Boulder, 1949).

\section{CRYSTALLOGRAPHY}

\section{Crystallographic Data for Urea Calcium Nitrate and Urea Cadmium Nitrate}

THE crystal structures of urea calcium nitrate and urea cadmium nitrate are being investigated as part of a survey of hydrogen bonding in urea inorganic salt complexes. Preliminary work has shown that urea calcium nitrate and urea cadmium nitrate are isostructural and belong to the monoclinic system.

By using small single crystals mounted on a Seifert diffractometer and copper $K_{\alpha}$ radiation, accurate values for the translations $d_{001}, d_{100}, d_{101}$ and $b$ were obtained. The accurate estimates were made by plotting the values of these translations, determined from the $00 l, h 00, h 0 l$ (for $h=1$ ) and $0 k 0$ reflexions, against the function $\frac{1}{2}\left(\frac{\cos ^{2} \theta}{\sin \theta}+\frac{\cos ^{2} \theta}{\theta}\right)$ and extrapolating to zero.

The monoclinic angle $\beta$ was calculated by means of the equation:

$$
\beta=\arccos \frac{d^{2}{ }_{101}\left(d^{2}{ }_{100}+d^{2}{ }_{001}\right)-\left(d_{100} d_{001}\right)^{2}}{2 d_{100} d_{001} d^{2}{ }_{101}}
$$

